Copyright C1997, American Institute of Aeronautics and Astronautics, Inc.

AIAA Meeting Papers on Disc, January 1997

A9715804, F49620-95-1-0364, AIAA Paper 97-0797

\title{
Dynamics of drop deformation and formation during secondary breakup in the bag breakup regime
}

\author{
W.-H. Chou \\ Michigan Univ., Ann Arbor \\ L.-P. Hsiang \\ Michigan Univ., Ann Arbor \\ G. M. Faeth \\ Michigan Univ., Ann Arbor
}

\begin{abstract}
AIAA, Aerospace Sciences Meeting \& Exhibit, 35th, Reno, NV, Jan. 6-9, 1997
Secondary drop breakup in the bag breakup regime was studied experimentally. The properties of the parent drop, as well as the properties of drops formed by secondary breakup, were measured as a function of time for shockwave-initiated disturbances in air at normal temperature and pressure. The test liquids included water, ethyl alcohol, and various glycerol mixtures to yield Weber numbers of 13-20, liquid/gas density ratios of 633-893, Ohnesorge numbers of $0.0043-0.0427$, and Reynolds numbers of 1550-2150. Measurements involved single- and double-pulse shadowgraphy and holography to yield the structure, size, and velocity of the parent drop, and the sizes and velocities of drops produced by secondary breakup, as a function of time during the breakup process. The parent drop undergoes significant deformation and lateral growth during breakup, forming a thin bag having a basal ring that is characteristic of the bag breakup regime. The ring contains roughly 60 percent of the initial drop volume and eventually yields drops having mean diameters of roughly 30 percent of the initial drop diameter by a Rayleigh breakup process; drop size variations of these ring drops increase with increasing Weber numbers due to the formation of large 'node' drops that are characteristic of the onset of the multimode breakup regime. (Author)
\end{abstract}


AIAA 97-0797

\title{
DYNAMICS OF DROP DEFORMATION AND FORMATION DURING SECONDARY BREAKUP IN THE BAG BREAKUP REGIME
}

\author{
W.-H. Chou, ${ }^{*}$ L.-P. Hsiang ${ }^{\dagger}$ and G. M. Faeth ${ }^{* *}$ \\ Department of Aerospace Engineering \\ The University of Michigan \\ Ann Arbor, MI 48109-2118, U.S.A.
}

\begin{abstract}
Secondary drop breakup in the bag breakup regime was studied experimentally. The properties of the parent drop, as well as the properties of drops formed by secondary breakup, were measured as a function of time for shockwave-initiated disturbances in air at normal temperature and pressure. The test liquids included water, ethyl alcohol and various glycerol mixtures to yield Weber numbers of 13-20, liquid/gas density ratios of 633-893, Ohnesorge numbers of 0.0043-0.0427 and Reynolds numbers of 1550-2150. Measurements involved single- and double-pulse shadowgraphy and holography to yield the structure, size and velocity of the parent drop, and the sizes and velocities of drops produced by secondary breakup, as a function of time during the breakup process. The parent drop undergoes significant deformation and lateral growth during breakup, forming a thin bag having a basal ring that is characteristic of the bag breakup regime. The ring contains roughly $60 \%$ of the initial drop volume and eventually yields drops having mean diameters of roughly $30 \%$ of the initial drop diameter by a Rayleigh breakup process; drop size variations of these ring drops increase with increasing Weber numbers due to the formation of large "node" drops that are characteristic of the onset of the multimode breakup regime. Breakup of the bag yields nearly monodisperse drops having diameters of roughly $4 \%$ of the initial

"Graduate Student Research Assistant.

†Post-Doctoral Research Fellow, currently with the Industrial Technology Research Institute, Taiwan

**Professor, Fellow AIAA.
\end{abstract}

drop diameter, due to Rayleigh-like breakup of the membrane-like bag. Velocity distributions of the drops formed by ring and bag breakup were individually independent of drop size but varied as a function of time and differed between the two groups. Many features of these phenomena were successfully correlated using phenomenological analysis. Finally, integrating drop size, drop velocity and drop formation rate properties over the period of breakup showed that the bag breakup process requires considerable time (5-6 characteristic breakup times) and extends over a considerable streamwise distance (30-60 initial drop diameters) by the end of breakup; this behavior implies that bag breakup should be treated as a rate process, rather than by jump conditions, in some instances.

\section{Nomenclature}

$\mathrm{C}_{\mathrm{D}} \quad=$ drop drag coefficient

$\overline{\mathrm{C}}_{\mathrm{D}} \quad=$ mean drop drag coefficient over a given time

$\mathrm{C}_{\mathrm{r}}=$ empirical constant

$\mathrm{d}=$ drop diameter

$\mathrm{d}_{\mathrm{bave}}=$ average diameter of bag

$\mathrm{d}_{\mathrm{bd}}=$ diameter of drops formed from bag

$\mathbf{d}_{\mathrm{bda}}=$ number averaged diameter of drops formed from bag

$\mathrm{d}_{\mathrm{bmax}}=$ maximum diameter of bag

$\mathrm{d}_{\mathrm{bmin}}=$ minimum diameter of bag

$d_{\max }=$ maximum dimension of object

$\mathbf{d}_{\min }=$ minimum dimension of object

$\mathrm{d}_{\mathrm{p}}=$ cross-stream diameter of parent drop

$d_{\mathrm{r}} \quad=$ tube diameter of ring

$\mathrm{d}_{\mathrm{nd}}=$ diameter of drops formed from the ring

$\mathrm{e}_{\mathrm{b}}=$ ratio of maximum/ minimum bag diameters, $\mathrm{d}_{\mathrm{bmax}} / \mathrm{d}_{\mathrm{bmin}}$ 


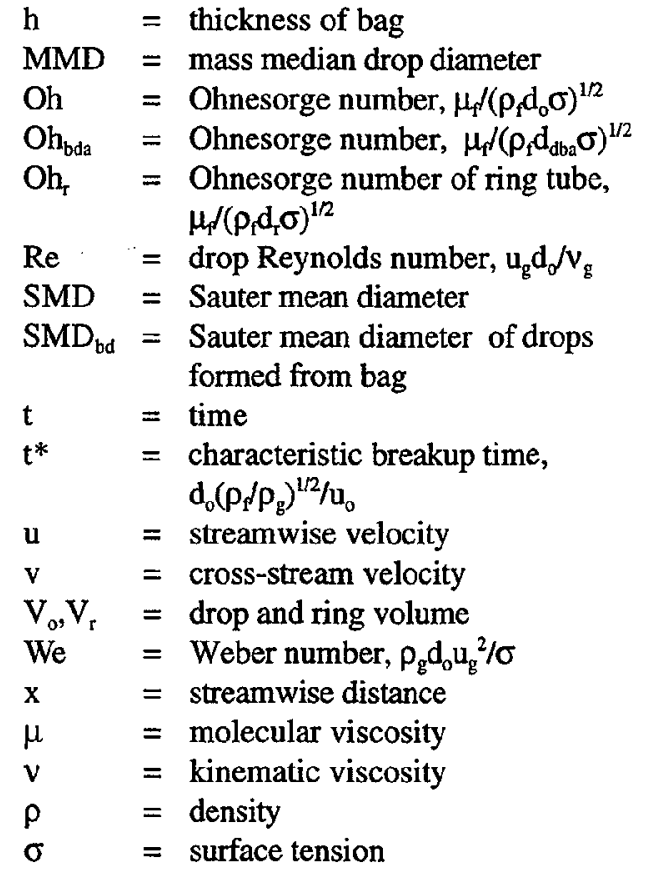

Subscripts

$$
\begin{array}{ll}
\mathrm{f} & =\text { liquid-phase property } \\
\mathrm{g} & =\text { gas-phase property } \\
\mathrm{p} & =\text { parent-drop property } \\
\mathrm{o} & =\text { initial property }
\end{array}
$$

\section{Introduction}

The secondary breakup of drops is an important fundamental process of sprays. In particular, drops formed by primary breakup are intrinsically unstable to secondary breakup, while secondary breakup can be the rate controlling process within dense sprays in much the same way that drop vaporization can be the rate controlling process within dilute sprays. ${ }^{1-4}$ Motivated by these observations, the objective of the present investigation was to extend earlier studies of the regimes and outcomes of secondary breakup due to shock-wave disturbances, ${ }^{5-8}$ to consider the evolution of both the properties and the rate of formation of drops resulting from secondary breakup as a function of time during bag breakup

Several recent summaries of available information about secondary breakup are available $;^{1-13}$ therefore, the following discussion of past investigations of secondary breakup will be brief. Shock-wave disturbances were considered during most earlier studies, providing a step change of the ambient environment of the drop, similar to conditions experienced by drops at the end of primary breakup. The main findings of initial work along the lines included the conditions required for particular deformation and breakup regimes, the times required for the onset and end of breakup, the drag properties of deformed drops, and the drop size and velocity distributions at the end of the breakup process (i.e., the jump conditions). An interesting feature of these results is that secondary breakup extended over appreciable regions of time and space and was not properly described by jump conditions in some instances. For example, Liang et al. ${ }^{14}$ show that breakup times are $5.5 \mathrm{t}^{*}$ (where $t^{*}$ is defined by Ranger and Nicholls ${ }^{16}$ as a characteristic secondary breakup time for shear breakup) for a wide range of drop conditions, which is comparable to flow residence times within the dense spray region where secondary breakup is a dominant process. ${ }^{1-4}$ Viewed another way, the original (or parent) drop moves roughly 40 initial drop diameters, while the smallest drops formed by secondary breakup move up to 100 initial drop diameters during the period of breakup for typical shear breakup processes. ${ }^{6-7}$ Such distances can represent a significant fraction of the length of the dense spray region. These observations suggest that the time-resolved features of secondary breakup eventually must be understood, i.e., the size and velocity distributions of the drops, and the rate at which liquid is removed the parent drop, must be known as a function of time during secondary breakup. Motivated by this observation, recent work in this laboratory has concentrated on studies of the temporal properties (dynamics) of particular secondary breakup processes.

The first phase of the study of the temporal properties of secondary breakup was limited to small Ohnesorge number conditions (Oh $<0.04$, where secondary breakup regime transitions are independent of drop liquid viscosity), to large values of $\rho_{\mathrm{f}} / \rho_{\mathrm{g}}\left(\rho_{\mathrm{f}} / \rho_{\mathrm{g}}>680\right.$, where gas-phase processes approximate quasisteady behavior) and to the shear breakup regime (where secondary breakup proceeds by stripping drop liquid from the periphery of the parent drop).$^{8} \quad$ It was found that the drop size distributions produced by secondary breakup satisfied Simmons' universal root normal 
distribution function with $\mathrm{MMD} / \mathrm{SMD}=1.2$ at each instant of time ${ }^{16}$ (see Belz ${ }^{17}$ for a general discussion of the properties of this distribution function); this is a helpful property because with the MMD/SMD ratio known, this two-parameter distribution function is fully defined by a single parameter, e.g., the SMD. The SMD of drops produced by shear breakup were independent of surface tension and exhibited transient and quasisteady regimes as a function of time that were related to the development of liquid flow within the parent drop due to the action of drag on its surface. The parent drop accelerated rapidly due to the large drag coefficient that was caused by drop deformation. The velocity distribution functions of drops produced by secondary breakup were uniform with mean drop velocities somewhat larger than the velocity of the parent drop at each instant of time and significant $\mathrm{rms}$ drop velocity fluctuations over the drop size distribution. The rate of liquid removal from the parent drop was correlated reasonably well by a clipped-Gaussian function which could accommodate both the onset and end of breakup. Finally, these properties, including the properties of the residual parent or drop-forming drop, were correlated reasonably well using phenomenological theories. ${ }^{6-8}$

The present study seeks to extend information about the temporal properties of secondary breakup from the shear breakup regime to the bag breakup regime, where secondary breakup proceeds by deformation of the center of the drop into a thin balloon-like bag that extends in the downstream direction from a thicker ringlike structure at its base, with both the bag and the ring subsequently dividing into drops. An understanding of bag breakup is important for two reasons: (1) the bag breakup regime bounds the region where drops only deform and do not break up, which provides fundamental clues about the mechanism of the onset of secondary breakup, and (2) the complex multimode breakup regime is bounded by the bag- and shear-breakup regimes which clearly must be understood before addressing the important multimode breakup mechanism. $^{5-7}$ Similar to past study of shear breakup, the present investigation emphasized new measurements of the temporal properties of bag breakup while developing approximate phenomenological theories in order to help interpret and correlate the measurements.
The present measurements were carried out using a shock tube facility, with the environment of the test drops during breakup roughly approximating air at normal temperature and pressure (NTP). Properties during breakup were observed using single- and double-pulse shadowgraphy and holography, to find the properties of the parent drop and both the size and velocity properties of drops produced by secondary breakup, as a function of time during breakup. Test conditions were limited to relatively large liquid/gas density ratios $\left(\rho_{\mathrm{f}} / \rho_{\mathrm{g}}>\right.$ 500 ), in order to minimize potential complications due to the inertia of the continuous phase, and relatively small Ohnesorge numbers (Oh $<0.1$ ), in order to minimize potential complications due to effects of liquid viscosity. As a result, the present test conditions are most representative of secondary breakup within sprays near atmospheric pressure. The experiments also were confined to the bag breakup regime. For present ranges of $\rho_{\mathrm{f}} / \rho_{\mathrm{g}}$ and Oh, this implies $13<\mathrm{We}<35$ in order to exceed criteria for the onset of the bag breakup regime but not to exceed the criteria for onset of the multimode breakup regime. ${ }^{5}$ Drop liquids included water, ethyl alcohol and various glycerol mixtures, in order to provide information about effects of drop liquid properties.

The paper begins with a description of experimental methods. Results are then discussed, considering visualization of the breakup process, the properties of the parent drop, the properties of drops formed from the basal ring of the bag, the properties of drops formed from bag itself and the overall properties of bag breakup, in turn.

\section{Experimental Methods}

\section{Apparatus}

The test apparatus will only be described briefly because it was similar to earlier work..$^{5-8}$ A shock tube with the driven section open to the atmosphere was used for the measurements, similar to the arrangement used by Ranger and Nicholls. ${ }^{15}$ The driven section had a rectangular cross-section ( $38 \mathrm{~mm}$ wide and $64 \mathrm{~mm}$ high) and a length of $6.7 \mathrm{~m}$, with the test location $4 \mathrm{~m}$ from its downstream end. This configuration 
provided test times of $17-21 \mathrm{~ms}$ in the uniform flow region behind the incident shock wave.

The test location had quartz windows (25 mm high and $305 \mathrm{~mm}$ long, mounted flush with the interior of the side walls) to allow observations of drop breakup. A vibrating capillary tube drop generator, similar to the arrangement described by Dabora, ${ }^{18}$ was used to generate a stream of drops having a constant diameter. Measurements of bag breakup properties require significant spacing between drops due to the large cross-stream dimensions of the bag and its basal ring; therefore, an electrostatic drop selection system was used to control the spacing between the drops, similar to the arrangement developed by Sangiovanni and Kestin. ${ }^{19}$ The resulting drop stream passed through $6 \mathrm{~mm}$ diameter holes in the top and bottom of the driven section, crossing the central plane of the driven section at the test location. Test drops had diameters less than $1 \mathrm{~mm}$, while the spacing between drops was 9-12 $\mathrm{mm}$; therefore, drops always were present within the region observed while interactions between adjacent drops during bag breakup were negligible. ${ }^{s}$

\section{Instrumentation}

Single- and double-pulsed shadowgraphy and holography were used to observe the properties of the parent drop and the size and velocity distribution functions of drops produced by secondary breakup, as a function of time during breakup. The arrangement was similar to Chou et al. ${ }^{8}$ with the shadowgraph system created by simply blocking the reference beam of the holocamera. The holocamera system involved two frequency doubled YAG lasers (Spectra Physics Model GCR-130, $532 \mathrm{~nm}$ wavelength, 7 ns pulse duration, up to $300 \mathrm{~mJ}$ per pulse) which could be controlled to provide pulse separations as small as 100 ns. An off-axis holocamera arrangement was used with the optics providing a $25 \mathrm{~mm}$ diameter field of view at the test drop location and a 3:1 primary magnification of the hologram image. The laser pulse times were sufficiently short to stop the motion of the drops on the film. Reconstruction of the double-pulse holograms yielded two images of the drop field caused by breakup so that drop velocities could be found given the time of separation between the pulses (which was measured with a digital oscilloscope). The second laser pulse was somewhat weaker than the first, which allowed directional ambiguity to be resolved because stronger pulses yield sharper reconstructed images.

The hologram reconstruction system was similar to the arrangement used by Chou et al. ${ }^{8}$ A helium-neon laser (Spectra Physics Model $124 \mathrm{~B}$, cw laser, $35 \mathrm{~mW}$ of optical power) was used to reconstruct the image. The reconstructed image was observed using a CCD camera (Sony, Model XC-77) with optics to yield a magnification of 300:1 and a field of view of the image (on the monitor) of $1.2 \times 1.4 \mathrm{~mm}$. The optical data was obtained using a frame grabber (Data Translation DT 2851) and stored on a personal computer (Micron, Pentium 90). The images were processed using Media Cybernetics Image-Pro Plus software. Various locations in the hologram reconstruction were observed by traversing the hologram in two directions, and the videocamera of the image display in the third direction. Positions were selected for viewing using stepping motor driven linear traversing systems (Velmex, Model VP9000) having $1 \mu \mathrm{m}$ positioning accuracies. The combined holocameral reconstruction system allowed objects as small as $3 \mu \mathrm{m}$ to be observed and objects as small as $5 \mu \mathrm{m}$ to be measured with $5 \%$ accuracy.

Drop sizes and velocities were measured in the same manner as earlier work. ${ }^{5.8}$ The diameters of mildly irregular objects were found by measuring their maximum and minimum diameters, $d_{\max }$ and $d_{\min }$, through the centroid of the image. Then assuming that the drop had an ellipsoidal shape, the drop diameter was taken to be equal to the diameter of a sphere having the same volume as the ellipsoid, e.g., $d^{3}=d^{2}{ }_{\min } d_{\max }$. More irregular objects were sized by finding the cross-sectional area and perimeter of the image and proceeding as before for an ellipsoid having the same properties. The velocity of each drop was found by measuring the distance between the centroid of its two images on a double-pulse hologram and dividing by the known time between laser pulses. Results at each condition were summed over at least four realizations, considering 100-200 liquid elements, in order to provide drop diameter and velocity correlations. Experimental uncertainties caused by the present 
definition of drop diameters are difficult to quantify, however, they are felt to be small in comparison to the accuracy of the size and distance measurements and sampling limitations. Estimated experimental uncertainties (95\% confidence) based on the latter effects are less than $10 \%$ for drop diameters and less than $15 \%$ for streamwise drop velocities.

\section{Test Conditions}

The test conditions are summarized in Table 1. Test drops of water, ethyl alcohol and various glycerol mixtures were used to provide a reasonably wide range of liquid properties within the low Ohnesorge regime. The liquid properties were obtained from Lange, ${ }^{20}$ except for the surface tensions of the glycerol mixtures which were measured in the same manner as $\mathrm{Wu}$ et al. ${ }^{21}$ The ranges of the test variables were as follows: $\mathrm{d}_{\mathrm{o}}=0.62-0.85 \mathrm{~mm}, \rho_{\mathrm{f}} / \rho_{\mathrm{g}}=633-893, \mathrm{Oh}=$ $0.0043-0.0427, \operatorname{Re}=1550-2150$ and $\mathrm{We}=13$ 20. The present We range is narrow, consistent with the range of the bag breakup regime, but this mechanism still is very important because it bounds conditions where secondary breakup does not occur. The $\mathrm{Re}$ range of the present experiments is higher than conditions where gas viscosity has a significant effect on drop drag properties, e.g. $C_{D}$ for spheres only varies in the range 0.4-0.5 for this Reynolds number range. ${ }^{22}$ Shock Mach numbers were relatively low, less than 1.04; therefore, the physical properties of the gas in the uniform flow region behind the shock wave were nearly the same as room air.

\section{$\underline{\text { Results and Discussion }}$}

\section{Description of Breakup Properties}

Visualization of Bag Breakup. Typical flash shadowgraphs of bag breakup for the present test conditions are illustrated in Fig. 1. These conditions involve a water drop in air subjected to a shock wave disturbance with $\mathrm{We}=$ 20 and $\mathrm{Oh}=0.0044$. The shock wave and its associated velocity disturbance are moving downward on the photographs. Shadowgraphs are shown at various normalized times during the breakup process, $t / t^{*}$, where $t^{*}$ is the characteristic breakup time of Ranger and Nicholls. ${ }^{15}$ The time $\operatorname{span} 0 \leq t / t^{*} \leq 5$ is illustrated; this period generally corresponds to the time required to complete bag breakup at small $\mathrm{Oh}$ (in the region where the value of $\mathrm{Oh}$ does not affect breakup regime transitions). ${ }^{5}$ These breakup times do not vary significantly for wide ranges of We although they tend to be slightly larger, $t / t^{*}=5.5$, for shear breakup at small Oh. ${ }^{5}$

The first three photographs of Fig. 1 correspond to the drop deformation period, $0 \leq$ $t / t^{*} \leq 2$, for bag breakup. In this period, the drop deforms from a sphere to a disk-like shape due to the static pressure distribution along the drop surface. In particular, there are larger static pressures near the upstream and downstream stagnation points and smaller static pressures where the gas accelerates over the periphery of the drops, which tends to squeeze the drop into its characteristic disk-like shape. This deformation to a disk-like shape causes a large increase of the drag of the drop due to both the increased area of the drop normal to the direction of relative motion and the larger drag coefficient of thin disks compared to spherical objects. ${ }^{5}$ The time period $2 \leq t / t^{*} \leq 3$ corresponds to the period of bag growth by deflection of the center of the disk in the streamwise direction; the condition shown in Fig. 1 at $t / t^{*}=3$ corresponds to the maximum bag size just prior to initiation of breakup of the bag itself. The bag begins to break up at its farthest downstream position and the circular breakup region of the bag propagates upstream toward the thicker ring at the base of the bag (the basal ring formed by the periphery of the disk). This process seems similar to Rayleigh breakup of the two-dimensional membrane (the bag) and yields relatively small drops. The bag breakup process occurs for the period $3 \leq t / t^{*} \leq 4$ so that the next figure shown at $t / t *=4$ corresponds to the end of bag breakup where just the basal ring remains. The basal ring generally does not have a completely uniform tubular diameter; instead, nodes of somewhat larger diameter than the tubular diameter form as shown in Fig. 1. These nodes appear to be related to the larger number of nodes formed by higher mode distortion of the bag that is observed in the multimode breakup regime. ${ }^{5}$ The ring retains its integrity in the period $4 \leq t / t^{*} \leq 5$ while the diameter of the tube axis of the ring, the diameter of the node drops and the diameter of the tube itself, change slowly. At the end of this period, the ring divides into drops, by a process that looks very much like Rayleigh breakup, to 
yield a necklace-like ring of relatively large drops, which completes the bag breakup process.

For $\mathrm{Oh}<0.043$ and We corresponding to bag breakup, the process remains qualitatively similar to Fig. 1. The main effect of increasing We for these conditions is to increase the number of node drops for the ring, and to obtain some node-like disturbances on the bag itself, both of which are anticipated as the complex multimode breakup regime is approached. For $\mathrm{Oh}>0.1$, however, the appearance of bag breakup begins to change so that long ligaments are formed, somewhat analogous to the long-ligament regime observed for shear breakup at large $\mathrm{Oh}^{8}$

The behavior of bag breakup in the long-ligament regime is illustrated in Fig. 2. These conditions involve a glycerol drop (75\% glycerin by mass in water) in air subjected to a shock wave disturbance with $\mathrm{We}=20$ and $\mathrm{Oh}=$ 0.135. As before, the motions of the shock wave, and its associated velocity disturbance, are directed from the top to the bottom of each photograph. The breakup process is seen to be qualitatively similar to Fig. 1 for $0 \leq t / t^{*} \leq 4$. At longer times, however, a new mode, similar to the modes seen in the multimode breakup regime, forms at the center of the bag (which corresponds to the center of the disk). The basal ring of the bag also develops node drops, and finally breaks up into a necklace-like ring of drops, but more slowly than at small Oh, e.g., final breakup is only completed at $t / t^{*}=8$ in Fig. 2, compared to $t / t^{*}=5$ in Fig. 1. In addition, the node drops retain portions of the ring or the bag as ligaments for a time unlike behavior at smaller $\mathrm{Oh}$ (the ligament associated with the center node is particularly prominent in Fig. 2). Similar to earlier considerations of the temporal properties of shear breakup, ${ }^{8}$ these complications of large ligaments were avoided for the present by only considering $\mathrm{Oh} \leq 0.1$.

Bag Breakup Parameters. In order to fix ideas concerning the properties of bag breakup properties, notation concerning the bag and its basal ring are identified in Fig. 3. This figure is an illustration of the deformed drop near the time when breakup of the downstream end of the bag begins, i.e., near $t / t^{*}=3$ for $\mathrm{Oh}<0.1$. At this condition, the bag has maximum and minimum diameters in the streamwise and cross-stream directions, $d_{b \max }$ and $d_{b \min }$, and a thickness $h$. The basal ring has a tubular diameter $d_{\mathrm{r}}$ and an outer diameter of the ring of $d_{p}$. The streamwise and cross-stream velocities of the tubular ring axis are $u_{p}$ and $v_{p}$, respectively, while the streamwise velocity of the ambient gas is $u_{g}$. Since $u_{g}>u_{p}$ throughout the breakup process, the presence of the bag causes the gas to stagnate near the ring; thus, the mean static pressure within the bag is greater than the local ambient static pressure, i.e., $p_{p}>p_{g}$, which provides the mechanism to inflate the bag. These parameters will be used to summarize the dynamic properties of the parent drop, the basal ring and the bag, and the overall properties of bag breakup, in subsequent sections.

\section{Parent Drop Properties}

Parent Drop Size. A composite figure giving the appearance of bag breakup as inset photographs, and the ratio $\mathrm{d}_{\mathrm{p}} / \mathrm{d}_{\mathrm{o}}$, as a function of $\mathrm{t} / \mathrm{t}^{*}$ during bag breakup is illustrated in Fig. 4 . The measurements shown on the figure are for water, ethyl alcohol and glycerol drops having We in the range $13-20$ and $\mathrm{Oh} \leq 0.043$. Finally, the time periods for various bag breakup processes, as discussed in connection with Figs. 1 and 2 , are indicated on the figure, as follows: $t / t^{*}$ of $0-2$, deformation period; $t / t^{*}$ of $2-3$, bag growth period; $t / t^{*}$ of $3-4$, bag breakup period; and $t / t^{*}$ of $4-5$, ring breakup period.

Consideration of the transition between a spherical drop, and a relatively thin disk aligned normal to the flow direction, in the deformation period $\left(0 \leq t / t^{*} \leq 2\right)$ has already been discussed in connection with Figs. 1 and 2. This deformation is caused by increased static pressures near the upstream and downstream stagnation points along the axis of the drop, combined with decreased static pressures near the drop periphery due to increased flow velocities in this region. This pressure distribution tends to squeeze the drops into a thin disk-like shape. A detailed analysis of this process was not undertaken; instead, the deformation process can be correlated reasonably well according to the following empirical correlation suitable for the present range of test conditions:

$\mathrm{d}_{\mathrm{p}} / \mathrm{d}_{\mathrm{o}}=1.0+0.5 \mathrm{t} / \mathrm{t}^{*}, \quad 0 \leq \mathrm{t} / \mathrm{t}^{*} \leq 2$ 
Subsequent consideration of parent drop size parameters will focus on the properties of the ring. This interest is motivated by the fact that the size of the ring ultimately controls the size of the drops formed by ring breakup while these drops tend to dominate the size properties of drops formed by bag breakup because they are the largest drops in the size distribution. In addition, subsequent considerations will show that the ring, and thus the drops formed from the ring, comprise a major fraction of the original volume of liquid in the parent drop.

The results illustrated in Fig. 4 show that the rate of lateral acceleration of the ring diameter is largest in the period where the bag is present, with subsequent accelerations progressively approaching small values toward the end of the bag breakup period. This behavior suggests that the higher pressure within the bag, caused by stagnation of the gas flow relative to the drop by the bag, discussed earlier, is mainly responsible for the outward acceleration of the ring, as well as growth of the bag. This pressure difference progressively disappears as the bag disappears so that the ring simply continues to coast outward in the latter stages of the breakup process; this behavior is supported by the relatively constant outward velocity of the ring diameter toward the end of breakup. These ideas are developed in the following to obtain the predicted variation of $\mathrm{d}_{\mathrm{p}} / \mathrm{d}_{\mathrm{o}}$ as a function of $t / \mathrm{t}^{*}$ for the period $2 \leq t / t^{*} \leq 6$ that is illustrated in Fig. 4.

Analysis of ring growth was carried out ignoring acceleration of the parent drop, i.e., it was assumed that the relative velocity of the ring with respect to the gas is equal to the initial relative velocity, $u_{g}$; the variation of the diameter of the tube axis of the ring was also neglected even through later considerations will show that this diameter decreases by almost a factor of two during the time period of interest; and circumferential surface tension forces were ignored due to the relatively large diameter of the ring at the start of the ring acceleration process. Other assumptions will be discussed as they are introduced. Considering the radial acceleration of the ring tube, conservation of momentum based on the notation and configuration illustrated in Fig. 3 yields:

$\rho_{\mathrm{f}}\left(\pi^{2} \mathrm{~d}_{\mathrm{p}} \mathrm{d}_{\mathrm{r}}^{2} / 4\right) \mathrm{d}^{2}\left(\mathrm{~d}_{\mathrm{p}} / 2\right) / \mathrm{dt}^{2}$

$$
=\mathrm{C}_{\mathrm{r}}\left(\rho_{\mathrm{g}} \mathrm{u}_{\mathrm{g}}^{2} / 2\right)\left(\pi \mathrm{d}_{\mathrm{p}} \mathrm{d}_{\mathrm{t}}\right)
$$

where $C_{r}$ is an empirical constant to account for the fact that the pressure difference across the ring is only a fraction of the ideal stagnation pressure increase due to effects of gas motion across the ring and the motion of the gas in the bag, particularly as bag breakup proceeds, etc. Equation (2) can be simplified by adopting $d_{p} / d_{0}$ and $t / t^{*}$ as normalized dependent and independent variables, as follows:

$$
\begin{array}{r}
\mathrm{d}^{2}\left(\mathrm{~d}_{\mathrm{p}} / \mathrm{d}_{\mathrm{o}}\right) / \mathrm{d}\left(\mathrm{t} / \mathrm{t}^{*}\right)^{2}=\left(4 \mathrm{C}_{\mathrm{r}} / \pi\right)\left(\mathrm{d}_{\mathrm{o}} / \mathrm{d}_{\mathrm{r}}\right), \\
2 \leq \mathrm{t} / \mathrm{t}^{*} \leq 4
\end{array}
$$

where the time interval of concern is the period when the bag (or at least a portion of it) is present and where the right-hand side of this equation is taken to be a constant under the assumptions of the present approximate analysis. The initial conditions for Eq. (3) were chosen to match the value of $d_{p} / d_{o}$ at $t / t^{*}=2$ from Eq. (1), while adjusting the initial outward velocity of the ring diameter to best fit the present measurements, as follows:

$$
t / t^{*}=2: \begin{aligned}
& \mathrm{d}_{\mathrm{p}} / \mathrm{d}_{\mathrm{o}}=2.0 \\
& \quad \\
& \mathrm{~d}\left(\mathrm{~d}_{\mathrm{p}} / \mathrm{d}_{\mathrm{o}}\right) / \mathrm{d}\left(\mathrm{t} / \mathrm{t}^{*}\right)=0.8
\end{aligned}
$$

Finally, integrating Eq. (3) subject to the initial conditions of Eq. (4), and adjusting the value of the constant on the right-hand side of Eq. (3) to best fit the present measurements, yields:

$$
\begin{array}{r}
\mathrm{d}_{\mathrm{p}} / \mathrm{d}_{\mathrm{o}}=0.25\left(\mathrm{t} / \mathrm{t}^{*}\right)^{2}-0.18\left(\mathrm{t} / \mathrm{t}^{*}\right)+1.43, \\
2 \leq \mathrm{t} / \mathrm{t}^{*} \leq 4
\end{array}
$$

which is the form that is plotted in Fig. 4. This result implies $C_{r} \approx 0.04$ in Eq. (3), which is reasonable in view of the residual motions of the gas within the bag (particularly toward the end of bag breakup) and the fact that the relative velocity of the parent drop with respect to the gas is only roughly $70-90 \%$ of the initial relative velocity during the period of bag growth and breakup.

Proceeding to the ring breakup period, it is assumed that the ring, and the ring drops that are formed by breakup of the ring, simply coast outward with a constant radial velocity. This behavior agrees with the observed variation of $d_{p}$ 
in this time period, and involves neglecting the relatively small drag forces on drop liquid elements in the radial direction. Finally, the value of $d_{\rho} / d_{o}$ at $t / t^{*}=4$ is matched to the results of Eq. (5) but the outward coasting velocity in the ring breakup period is re-optimized to best fit the measurements. The final variation of $\mathrm{d}_{\mathrm{p}} / \mathrm{d}_{\mathrm{o}}$ in the ring breakup period then becomes:

$\mathrm{d}_{\mathrm{p}} / \mathrm{d}_{\mathrm{o}}=1.79\left(\mathrm{t} / \mathrm{t}^{*}\right)-2.51,4 \leq \mathrm{t} / \mathrm{t}^{*} \leq 6$

which is the form that is plotted in Fig. 4.

Taken together, Eqs. (1), (5) and (6) are seen to provide a reasonable correlation of the measured variation of $d_{p} / d_{o}$ as a function of $t / t^{*}$ in Fig. 4. These results suggest that the flow resistance caused by the bag, and the remaining portions of the bag during its breakup period, are mainly responsible for the cross-stream spread of drops formed by breakup of the ring. Stabilization of this motion by surface tension in the deformation period can be important; after all, this mechanism is responsible for controlling drop defor-mation and preventing drop breakup in the deformation regime which is present at We slightly smaller than the bag breakup regime. Nevertheless, effects of surface tension on the radial dispersion of liquid during bag breakup appears to be relatively small.

Parent Drop Velocity. The motion of the parent drop relative to the gas is plotted as a function of normalized time in Fig. 5. The various breakup periods - deformation, bag growth and ring growth - are marked on the plot for reference purposes. It should be noted, however, that these breakup period designations are only appropriate for present test conditions.

The parent drop exhibits considerable acceleration during the breakup period, similar to past observations of the motion of parent drops for shear breakup..$^{5-8}$ In fact, the absolute and relative velocities of the parent drop are comparable at the end of the ring growth period, which implies a reduction of the relative velocity of he parent drop of roughly $50 \%$ during the time of breakup, which is quite substantial. This behavior comes about due to both growth in the cross-stream dimensions of the parent drop, as a result of deformation and bag formation, as well as increased drag coefficients of the deformed parent drop compared to spherical objects.

The measurements illustrated in Fig. 5 were replotted so that average drag coefficients could be determined for the various breakup periods. These drag coefficients were averaged over the particular breakup period using all available test data.

In order to provide a common basis for comparing drag effects during various breakup periods, the average drag coefficients were normalized using the original cross-sectional area of the undeformed drop and the initial relative velocity of the drop with respect to the ambient gas. Thus, the ratio of these drag coefficients to the drag coefficients of spheres at similar Reynolds numbers, $\bar{C}_{D}=0.4-0.5$, represents the ratio of the drag forces on the present drop compared to an undeformed sphere. The resulting mean drag coefficients are as follows: $\overline{\mathrm{C}}_{\mathrm{D}}=1.9$ for $0 \leq t / t^{*} \leq 2$ in the deformation period, $\overline{\mathrm{C}}_{\mathrm{D}}=$ 6.2 for $2 \leq t / t^{*} \leq 3$ in the bag growth period, and $\bar{C}_{D}=4.1$ for $3 \leq t / t^{*} \leq 5$ in the ring growth and breakup periods. Notably, all these drag coefficients are significantly larger than $\bar{C}_{D}=0.4-$ 0.5 which would be appropriate for spheres over the same range of Reynolds numbers as the present experiments. ${ }^{22}$ The fact that the $\bar{C}_{D}$ are largest in the bag growth period is representative of the large cross-stream dimensions of the bag and its basal ring. The reduced drag of the ring growth and breakup periods is then representative of the lost flow resistance when the bag is no longer present. Finally, the average drag coefficients of the deformation period are consistent with earlier results, ${ }^{5-8}$ after accounting for differences in their values due to different conventions for the cross-sectional areas and the relative velocities of the parent drop.

\section{Basal Ring Properties}

Ring Volume. Drop sizes formed from the bag and the basal ring of the bag are substantially different; therefore, it is important to know the relative volumes of the bag and its basal ring in order to estimate drop sizes produced by bag breakup. Thus, measurements were 
undertaken to establish the distribution of parent drop liquid between the bag and the basal ring over the complete range of the present data. These measurements were made by characterizing the ring at the end of bag breakup, including the volume of the nodal drops as well as the cylindrical sections of the ring in the region between the nodal drops.

The ratio of the liquid volume in the basal ring to the initial volume of the parent drop, $V_{r} / V_{o}$, is plotted as a function of $O h$ in Fig. 6. For present test conditions, each value of Oh corresponds to a particular drop liquid; nevertheless, it can be seen that $V_{r} / N_{0}$ is essentially independent of $\mathrm{Oh}$ over the present test range and yields an average value of this ratio of $56.1 \%$ with a standard deviation of $3.7 \%$. Lane ${ }^{23}$ carried out early measurements of bag breakup and mentions a determination of $\mathrm{V}_{\mathrm{r}} / \mathrm{V}_{\mathrm{o}}=$ 75\%. Nevertheless, this earlier value is only mentioned in passing with no information provided about its accuracy and method of determination; therefore, the reliability of the earlier value is uncertain.

Tube Axis Diameter. Given that the liquid volume of the ring is a fixed fraction of the initial drop volume, it should be possible to determine the diameter of tube axis of the ring as a function of the ring diameter. In particular,

$\mathrm{V}_{\mathrm{r}} / \mathrm{V}_{\mathrm{o}}=\left(\pi^{2} \mathrm{~d}_{\mathrm{p}} \mathrm{d}_{\mathrm{r}}^{2} / 4\right) /\left(\pi \mathrm{d}_{\mathrm{o}}^{3} / 6\right)$

Thus,

$$
\begin{aligned}
\mathrm{d}_{\mathrm{l}} / \mathrm{d}_{\mathrm{o}} & =\left(2 \mathrm{~V}_{\mathrm{N}} /\left(3 \pi \mathrm{V}_{\mathrm{o}}\right)\right)^{1 / 2} /\left(\mathrm{d}_{\mathrm{p}} / \mathrm{d}_{\mathrm{o}}\right)^{1 / 2} \\
& =0.35 /\left(\mathrm{d}_{\mathrm{p}} / \mathrm{d}_{0}\right)^{1 / 2}
\end{aligned}
$$

where $d_{p} / d_{o}$ is known as a function of time, $t / t^{*}$, from Fig. 4 or from Eqs. (1), (5) and (6).

Present measurements of $d_{r} / d_{0}$ are plotted as a function of $t / t^{*}$ in Fig. 7. The predictions of $d_{\mathrm{f}} / \mathrm{d}_{\mathrm{o}}$ from Eq. (8), using Eqs. (1), (5) and (6) to find $\mathrm{d}_{\mathrm{p}} / \mathrm{d}_{\mathrm{o}}$, are also shown on the plot for comparison with the measurements. There is significant scatter of the measurements due to problems of observing the ring, particularly when the bag is present. Nevertheless the measurements are seen to be in reasonably good agreement with the predictions, supporting a progressive but slow reduction of $\mathrm{d}_{\mathrm{l}} / \mathrm{d}_{\mathrm{o}}$ with increasing time due to the increased diameter of the tube axis of the ring.

Ring Drop Diameters. Two types of drops are formed from the ring, nodal drops and drops from the cylindrical portions of the ring between the nodes that are somewhat smaller than the nodal drops. The drops formed from the cylindrical portion of the ring are not subject to strong strain and appear to be the result of a classical Rayleigh breakup process of a nearly constant-diameter liquid column. In addition, the Ohnesorge numbers of the rings observed during the present investigation were relatively small $\left(\mathrm{Oh}_{\mathrm{r}}<0.11\right)$ so that effects of liquid viscosity should be small as well. Under these circumstances, the ratio of the diameter of the drops formed by ring breakup, and the ring diameter, should be a constant, as follows: ${ }^{29}$

$\mathrm{d}_{\mathrm{r} d} / \mathrm{d}_{\mathrm{r}}=1.88, \quad$ predicted

The Rayleigh breakup condition of Eq. (9) was evaluated using the present measurements. In doing this, the complication of the node drops was ignored (they will be considered later) and only drops formed from the intervening constantdiameter portions of the ring were considered. In addition, $\mathrm{d}_{\mathrm{t}}$ was determined for this expression at the time of ring drop breakup, i.e., $t / t^{*}=5$ where $\mathrm{d}_{\mathrm{r}} / \mathrm{d}_{\mathrm{o}}=0.15$ from Fig. 7.

The values of $d_{n d} / d_{1}$ measured during the present investigation are plotted as a function of Oh in Fig. 8, along with the Rayleigh breakup prediction of Eq. (9). As before, present experiments involved a nearly constant $\mathrm{O}$ (and $\mathrm{Oh}_{\mathrm{r}}$ ) for each liquid because the variation of We was small. The measurements do not suggest a significant effect of $\mathrm{Oh}$ over the present test range and yield

$\mathrm{d}_{\mathrm{rd}} / \mathrm{d}_{\mathrm{r}}=2.2, \quad$ measured

Clearly, Eq. (10) is in reasonably good agreement with the Rayleigh breakup prediction at small $\mathrm{Oh}$ given by Eq. (9), supporting Rayleigh breakup as the mechanism producing drops from the ring. An important complication of the Rayleigh breakup process, however, involves the larger node drops; in particular, the mechanism of their appearance still must be explained and they clearly merit further study. Another open issue 
is the time required for the Rayleigh breakup of the ring and how this time compares with the Rayleigh breakup times of other liquid columns, as discussed by McCarthy and Molloy. ${ }^{25}$ Finally, given $d_{\mathrm{r}} / d_{o}=0.15$ from Fig. 10, Eq. (10) implies $d_{x d} d d_{o}=0.33$ with the node drops being somewhat larger.

\section{Bag Properties}

Bag Dimensions. The bag is one of the most distinctive features of the bag breakup process and was studied in order to gain insight about bag breakup dynamics and jump conditions. The first issue that was addressed was the size of the bag at the end of the bag growth period. This issue is important because given that $V_{r} / V_{0}$ is nearly constant, the volume of the bag liquid is correspondingly known and combined with information about the surface area of the bag provides a first estimate of the bag thickness and thus the probable size of drops formed by breakup of the bag.

The definitions of bag dimensions have been discussed in connection with Fig. 3. The present measurements of the major and minor dimensions of the bag were made just prior to the onset of bag breakup, to yield $d_{b a v e}$ and the ratio, $e_{b}$. These properties are plotted as a function of Oh for present measurements in Fig. 9. Similar to other properties of bag breakup, however, these properties do not vary significantly with Oh for the present low $\mathrm{Oh}$ conditions. Thus, averaging over the entire data base, the following average values were obtained:

$\mathrm{d}_{\mathrm{bave}} / \mathrm{d}_{\mathrm{o}}=3.58, \quad \mathrm{e}_{\mathrm{b}}=1.21$

The values provided in Eq. (11) are plotted on Fig. 9 for comparison with the measurements. These simple expressions are seen to provide reasonably good correlations of the measured bag geometry parameters.

The final bag property that was estimated was the bag thickness. This estimate was based on the assumption of a uniform bag thickness, $h$, a relatively thin bag, $h / d_{\text {bave }} \ll 1$, with the bag taken to be a sphere having a diameter of $d_{\text {bave, }}$, while ignoring the absence of bag surface due to the presence of the basal ring and its open area. Under these assumptions, the ratio of the bag to the initial drop volume can be expressed as follows:

$\left(V_{o}-V_{r}\right) / V_{o}=\pi d_{\text {bave }}^{2} h /\left(\pi d_{o}{ }^{3} / 6\right)$

Then simplifying and rearranging, Eq. (12) yields the following expression for the normalized bag thickness:

$\mathrm{h} / \mathrm{d}_{\mathrm{o}}=\left(\mathrm{V}_{\mathrm{o}}-\mathrm{V}_{\mathrm{t}}\right)\left(\mathrm{d}_{\mathrm{o}} / \mathrm{d}_{\mathrm{bave}}\right)^{2} /\left(6 \mathrm{~V}_{\mathrm{o}}\right)$

Based on the present determinations of $V_{P} / V_{0}$ and $\mathrm{d}_{\text {bave }} / \mathrm{d}_{\mathrm{o}}$ given in Figs. 6 and 9 , the present estimate $\mathrm{h} / \mathrm{do} \approx 0.6 \%$. In view of the rather blunt shape of the bags, however, this estimate probably is small with a value of $h / d_{o}$ of $1 \%$ being more representative. Additional information about $\mathrm{h} / \mathrm{d}_{\mathrm{o}}$ will be discussed next, in connection with consideration of the drop sizes formed by breakup of the bag.

Bag Drop Diameter. The properties of drops formed by breakup of the bag, along with a few determinations of bag thickness by measurements from holograms, are summarized in Table 2. It should be noted that the values of $h$ given in Table 2 are not very reliable at the present time, they approach present limits of spatial resolution and involve additional problems of estimating film thicknesses from the region where the bag breaks up into drops. In view of these problems, it is estimated that the values of $h$ in Table 2 might be too large by as much as a factor of two. Current work is seeking to reduce these uncertainties about $h$, although corresponding drop diameter measurements for drops found from bag breakup are felt to be reliable within the uncertainties stated earlier. Entries provided in Table 2 include $d_{0}$, the time when drop sizes were measured (except for one condition at $t / t^{*}=3$, these results were averaged over the entire breakup period $t / t$ * $=3-4$ ), the number-averaged bag drop diameter, $\mathrm{d}_{\mathrm{bda}}$, and the Sauter mean diameter, $S \mathrm{MD}_{\mathrm{bd}}$, of drops formed from the breakup of the bag, along with several normalizations of these properties.

Comparing mean drop diameters at the start of bag breakup and averaged over the entire bag breakup period for glycerol (42\%) indicates an increase of the drop sizes as the basal ring of the bag is approached. This is not unexpected as some stretch of the bag membrane, and 
corresponding reduction of size of drops formed from the membrane, is expected. Nevertheless, the variation is not large with drops formed initially being only $15 \%$ smaller than the mean drop size.

A second issue of interest about drops formed by breakup of the bag itself is the variation of mean drop sizes with $\mathrm{Oh}$. The results of Table 2 show that both $\mathrm{d}_{\mathrm{bav}} / \mathrm{d}_{0}$ and $\mathrm{SMD}_{\mathrm{bd}} / \mathrm{d}_{0}$ increase as $\mathrm{Oh}$ increases over the test range. Characterizing this behavior by the Ohnesorge number based on the average size of drops formed from the bag, it is seen that $d_{b d a} / d_{0}$ increases from $3.5 \%$ to $4.9 \%$ as $\mathrm{Oh}_{\mathrm{bda}}$ increases from 0.023 to 0.091 . This behavior suggests an effect of liquid viscosity on bag properties, and thus on the properties of drops formed from the bag; such behavior is not surprising in view of past observations of strong effects of liquid viscosity on the drop sizes formed by secondary breakup..$^{5-8}$

Mean drop sizes resulting from breakup of the bag vary somewhat with initial $\mathrm{Oh}$ as just noted, but yield an average value of $\mathrm{d}_{\mathrm{bd}} / \mathrm{d}_{\mathrm{o}}$ of $4 \%$, over the present test range. Thus, bag drops generally are relatively small and do not have as strong an effect on spray transport properties as the drops produced by breakup of the ring. For example, based on the diameter-squared behavior that tends to dominate drop properties in sprays, the lifetime of drops formed from the ring would be nearly 60 times longer than the lifetime of drops formed from the bag. Another issue concerning mean drop sizes is that $\mathrm{SMD}_{b d}$ and $d_{b d a}$ are nearly the same, e.g., the average value of the ratio $S M D_{b d} / d_{b d a}=0.89$. This behavior implies a nearly monodisperse size distribution for these drops, a property that will be considered in more detail next.

The size distribution function of drops formed by breakup of the bag is illustrated in Fig. 10. These results are plotted according to the root normal distribution function that has proven to be successful for a variety of drop and spray breakup processes.' Results for variations (MMD/SMD) $)_{\text {bd }}$ are shown on the plot for comparison with the measurements. In the past, this distribution function with MMD/SMD = 1.20 has been successful for correlating drop size distributions in sprays. The bag drops themselves, however, while correlating reasonably well according to the root normal distribution function, do so only with a much smaller value of $(\mathrm{MMD} / \mathrm{SMD})_{b d}=1.04$. As discussed earlier, however, this result is not unexpected due to the nearly monodisperse size distribution of drops formed from the bag because the bag membrane itself appears to have a relatively uniform thickness. The behavior of the drop size distribution function changes when drops formed from both the bag and the ring are considered, however, as discussed next in connection with overall breakup properties.

\section{Overall Breakup Properties}

Drop Size Distributions. Past work yielded different observations about overall drop size distributions resulting from bag breakup with Hsiang and Faeth ${ }^{5,6}$ finding drop size distribution functions represented reasonably well by the universal root normal size distribution function while Gel' fand et al. ${ }^{26}$ report a bimodal drop size distribution function with one nearly monodisperse group associated with drops formed from the ring and a second nearly monodisperse group associated with drops formed from the bag. The overall drop size distribution function properties were studied during the present investigation but it was found that in spite of the nearly monodisperse drops formed from the bag no bimodal behavior for the drop size distribution function was evident. Thus, present results concerning the drop size distribution function were correlated in terms of Simmons' root normal distribution function. ${ }^{16}$

The drop size distribution results for the present measurements of bag breakup properties are plotted in terms of the universal root normal distribution function in Fig. 11. These results are preliminary in that behavior over the entire test range has been emphasized rather more statistically significant results at a fewer number of conditions. Thus, the measurements illustrated in Fig. 11 are scattered due to inadequate statistics. In particular, bag breakup of individual drops yields a relatively small number of large drops that dominate the size distribution function because they represent a large fraction of the drop volume produced by breakup. Current work is emphasizing additional drop measurements in order to reduce the scatter. The results shown in Fig. 11, however, were 
best represented by the root normal distribution function with $\mathrm{MMD} / \mathrm{SMD}=1.2$, which is similar to earlier findings for other spray breakup processes."

Information about drop velocity distributions obtained thus far suggest that drops produced by bag breakup have velocities that are relatively independent of drop sizes and approximate the velocity of the parent drop at each instant of time (these velocities can be estimated from the results plotted in Fig. 5). The temporal and spatial range of bag breakup was estimated based on this information as discussed next.

The spatial and temporal properties of bag breakup based on the velocity results of Fig. 5 are illustrated in Fig. 12. These findings involve the normalized streamwise position of the drops, $\mathrm{x} / \mathrm{d}_{0}$, as a function of normalized time after the start of breakup, $t / t^{*}$. Results are shown for the parent drop (which is slowest to relax toward gas velocities) and for the most remote drop (which is the first drop formed from breakup of the bag and responds relatively rapidly to the gas motion due to its relatively small size). The most remote drop separates from the parent drop at $t / t^{*}$ $=3$ when the bag begins to break up. The breakup process itself typically is ended when breakup of the ring is completed, which occurs roughly at $\mathrm{t} / \mathrm{t}^{*}=5.5$ for present test conditions. In the coordinate system of Fig. 12, there is a small effect of $\rho_{\mathrm{d}} / \rho_{\mathrm{g}}$ on drop motion; therefore, results at the limits of the present test range, $\rho_{\mathrm{f}} / \rho_{\mathrm{g}}=630$ and 890 , have been illustrated on the plot.

The results illustrated in Fig. 12 indicate that the temporal and spatial ranges of bag breakup are comparable to earlier findings for shear breakup. ${ }^{8}$ In particular, the breakup period requires $t / t^{*}$ in the range $0-5.5$; in this period, the most remote drop moved a streamwise distance of roughly 60 initial drop diameters and the parent drop moves a streamwise distance of roughly 30 initial drop diameters. Finally, the results plotted in Fig. 4 imply that the largest drops formed by breakup of the ring spread laterally to a diameter of roughly 7 initial drop diameters. These times and distances are comparable to characteristic times and distances associated with the dense region of pressureatomized sprays; ${ }^{1}$ therefore, bag breakup similar to shear breakup, should be treated as a rate process rather than by jump conditions, in many instances.

\section{Conclusions}

The properties of the parent drop, as well as the properties of drops formed by secondary breakup, were measured as a function of time for bag breakup due to shock-wave initiated disturbances in air at normal temperature and pressure. The test liquids included water , ethyl alcohol and various glycerol mixtures to yield We of $13-20, \rho_{f} / \rho_{\mathrm{g}}$ of $633-893$, Oh of $0.0043-0.0427$ and Re of 1550-2150. The major conclusions of the study are as follows:

1. The basal ring formed from the parent drop contains roughly $60 \%$ of the initial drop volume and eventually yields drops having mean diameters of roughly $30 \%$ of the initial drop diameter due to a Rayleigh-like breakup process of the ring that occurs relatively abruptly near $\mathrm{t} / \mathrm{t}^{*}=5$.

2. The bag formed from the parent drop contains roughly $40 \%$ of the initial drop volume and eventually yields drops having mean diameters of roughly $4 \%$ of the initial drop diameter due to a Rayleigh-like breakup process of the membranelike bag. This breakup process propagates progressively from the tip to the basal ring end of the bag over the period $3 \leq t / t^{*} \leq 4$ and yields a nearly monodisperse drop size distribution; this behavior suggests a relatively uniform bag thickness of roughly $2-3 \%$ of the initial drop diameter.

3. Combining the populations of the drops formed from the basal ring and from the bag yields a drop size distribution that roughly satisfies the universal root normal distribution function with $\mathrm{MMD} / \mathrm{SMD}=1.2$ proposed by Simmons, ${ }^{16}$ similar to many other drop breakup processes including earlier observations for bag breakup. ${ }^{5}$ This behavior follows in spite of the nearly monodisperse drop size distribution of drops formed from the bag due to the relatively large volume of the basal ring and the size variation of drops formed from the ring due to the presence of relatively large node drops at various points along the ring at the time of ring breakup. 
4. Drop velocity distributions at each instant of time during breakup are relatively independent of the size of drops formed at each instant, although there is a considerable variation of mean drop velocities as a function of time as well as a significant difference of velocity between drops formed from the ring and from the bag due to their large size differences. Phenomenological analyses provided the basis for reasonably good correlations of drop velocities similar to earlier considerations of drop velocities for jump conditions. $^{5-8}$ Finally, the parent drop experiences large acceleration rates due to the development of both large cross-sectional areas and large drag coefficients due to deformation and bag formation.

5. The bag breakup process causes significant temporal and spatial dispersion of drops during the breakup period, as follows: the breakup process requires a total time of $t / t^{*}=5$; the cross-stream dispersion, based on the diameter of the ring axis when ring breakup is completed, amounts to roughly 7 initial drop diameters; and the streamwise dispersion involves a streamwise motion of the parent drop of roughly 30 initial drop diameters and of the most remote drop of roughly 60 initial diameters. These times and distances are not always small in comparison to characteristic times and distances of dense spray processes, implying that bag breakup should be treated as a rate process, rather than by jump conditions, in some instances, similar to shear breakup. ${ }^{8}$

\section{Acknowledgments}

This research was sponsored by the Air Force Office of Scientific Research Grant No. F49620-95-I-0364, under the technical management of J. M. Tishkoff. The authors would like to thank C. W. Kauffman for the loan of the shock tube facility and advice concerning its operation. The U.S. Government is authorized to reproduce and distribute copies of this article for governmental purposes notwithstanding any copyright notation thereon.

\section{$\underline{\text { References }}$}

${ }^{1}$ Faeth, G.M., "Spray Combustion Phenomena," Twenty-Sixth Symposium
(International) on Combustion, The Combustion Institute, Pittsburgh, in press.

${ }^{2}$ Faeth, G.M., Hsiang, L.-P. and Wu, P.-K., "Structure and Breakup Properties of Sprays," Int. J. Multiphase Flow, Vol. 21, Suppl., 1995, pp. 99-127.

${ }^{3}$ Wu, P.-K., Hsiang, L.-P. and Faeth, G.M., "Aerodynamic Effects on Primary and Secondary Breakup," Prog.Astro. Aero. Vol. 169, 1995, pp. 247-279.

${ }^{4}$ Faeth, G.M., "Structure and Atomization Properties of Dense Turbulent Sprays," TwentyThird Symposium (International) on Combustion, The Combustion Institute, Pittsburgh, 1990, pp. 1345-1352.

${ }^{5}$ Hsiang, L.-P. and Faeth, G.M., "NearLimit Drop Deformation and Secondary Breakup," Int. J. Multiphase Flow, Vol. 18, 1992, pp.635-652.

${ }^{6}$ Hsiang, L.-P. and Faeth, G.M., "Drop Properties After Secondary Breakup," Int. J. Multiphase Flow, Vol. 19, 1993, pp.721-735.

${ }^{7}$ Hsiang, L.-P. and Faeth, G.M. "Drop Deformation and Breakup Due to Shock Wave and Steady Disturbances," Int. J. Multiphase Flow, Vol. 21, 1995, pp.545-560.

${ }^{8}$ Chou, W.-H., Hsiang, L.-P. and Faeth, G.M. "Temporal Properties of Secondary Drop Breakup in the Shear Breakup Regime," Int. J. Multiphase Flow, submitted.

${ }^{9}$ Giffen, E. and Muraszew, A., The Atomization of Liquid Fuels, Chapman \& Hall, London, 1953.

${ }^{10}$ Hinze, J.O., "Fundamentals of the Hydrodynamic Mechanism of Splitting in Dispersion Processes," AIChE J., Vol. 1, 1955 , pp. 289-295.

${ }^{11} \mathrm{Clift}, \mathrm{R}$, Grace, J.R. and Weber, M.E., Bubbles, Drops and Particles, Academic Press, New York, 1978, pp. 26 and 339-347. 
${ }^{12}$ Krzeczkowski, S.A., "Measure-ment of Liquid Droplet Disintegration Mechanisms," Int. J. Multiphase Flow, Vol. 6, 1980, pp. 227239.

${ }^{13}$ Wierzba, A. and Takayama, K., "Experimental Investigations on Liquid Droplet Breakup in a Gas Stream," Report Inst. High Speed Mech., Tohoku Univ., Vol. 53, 1987, pp. $1-99$.

\footnotetext{
${ }^{14}$ Liang, P.Y., Eastes, T.W. and Gharakhari, A., "Computer Simulations of Drop Deformation and Drop Breakup," AIAA Paper No. 88-3142, 1988.
}

${ }^{15}$ Ranger, A.A. and Nicholls, J.A., "The Aerodynamic Shattering of Liquid Drops," AIAA I., Vol. 7, 1969, pp. 285-290.

${ }^{16}$ Simmons, H.C., "The Correlation of Drop-Size Distributions in Fuel Nozzle Sprays," J. Engr. for Power, Vol. 99, 1977, pp. 309-319.

${ }^{17}$ Belz, M.H., Statistical Methods in the Process Industries, Wiley, New York, 1973, pp. 103-104.

"Dabora, E.K., "Production of Monodisperse Sprays," Rev. Scient. Instrum. Vol. 38, 1967, pp. 502-506

${ }^{19}$ Sangiovanni, J. and Kestin, A.S., "A Theoretical and Experimental Investigation of the Ignition of Fuel Droplets," Combust. Sci. Technol., Vol. 16, 1977, pp. 59-70.
${ }^{20}$ Lange, N.A., Handbook of Chem-istry, 8th ed., Handbook Publishers, Inc., Sandusky, Ohio, 1952, pp. 1134 and 1709.

${ }^{2 ! W u, ~ P .-K ., ~ R u f f, ~ G . A . ~ a n d ~ F a e t h, ~ G . M ., ~}$ "Primary Breakup in Liquid/Gas Mixing Layers for Turbulent Liquids," Atom. Sprays, Vol. 1 , 1991, pp. 421-440.

22White, F.M., Viscous Fluid Flow, McGraw-Hill, New York, 1974.

${ }^{23}$ Lane, W. R., "Shatter of Drops in Streams of Air," Ind. Engr. Chem., Vol. 43, 1951, pp. 1312-1317.

${ }^{24}$ Dombrowski, H. and Hooper, P.C., "The Effect of Ambient Density on Drop Formation in Sprays," Chem. Engr. Sci., Vol. 19, 1962, pp. 29l-305.

${ }^{25}$ McCarthy, M.J. and Malloy, N.A., "Review of Stability of Liquid Jets and the Influence of Nozzle Design," Chem. Engr. I. Vol. 7, 1974, pp. 1-20.

${ }^{26}$ Gel'fand, B.E, Gubin, S.A. and Kogarko, S.M., "Various Forms of Drop Fractionation in Shock Waves and Their Special Characteristics," Inzh.Fiz.Zh., Vol. 27, 1974, pp. 119-126.

Tabie 1. Summary of the essi conditions"

\begin{tabular}{|c|c|c|c|c|c|c|c|}
\hline Liquid & $\begin{array}{c}d_{k} \\
(\mu m)\end{array}$ & $\begin{array}{c}\rho_{\mathrm{l}} \\
\left(\mathrm{kg} / \mathrm{m}^{3}\right)\end{array}$ & $\begin{array}{c}\rho_{d} / \rho_{R} \\
(\cdot)\end{array}$ & $\begin{array}{l}\mu_{\mathrm{r}} \times 10^{4} \\
(\mathrm{~kg} / \mathrm{ms})\end{array}$ & $\begin{array}{l}\sigma \times 10^{1} \\
(\mathrm{~N} / \mathrm{m})\end{array}$ & Oh $\times 10^{1}$ & $\begin{array}{l}\mathrm{Rc} \\
(-)\end{array}$ \\
\hline Waicr & 620 & 997 & 755 & 8.94 & 70.8 & 4.3 & $1670-1910$ \\
\hline Ealiyl alcohol & 630 & 800 & 633 & 16.0 & 24.0 & 15.0 & $1830-2080$ \\
\hline Glycciol $(2 \mid \%)$ & 650 & 1050 & 806 & 16.0 & 67.3 & 7.5 & $1550-1660$ \\
\hline Glyccrol (42\%) & 650 & 1105 & .857 & 35.0 & 65.4 & 16.1 & $1550-1910$ \\
\hline Glyccrol $(63 \%)$ & 850 & 1162 & 893 & 108.0 & 64.8 & 42.7 & 18.50 .2150 \\
\hline
\end{tabular}

"Bag breakup for $\mathrm{W}_{\mathrm{c}}=13-20$ in air inicially at $98.8 \mathrm{kPa}$ and $298 \pm 2 \mathrm{~K}$ in the driven section of the shonck lube with shock Mach numbers of 1.01-1.04, Propertics of air taken for condilions downsurcan of shock wave: $p_{\mathrm{g}}$ of $119.7-129.8 \mathrm{kPa}, p_{\mathrm{g}}$ of $1.25 \cdot 1.31 \mathrm{~kg} / \mathrm{m}^{3}$ and $\mu_{\mathrm{g}}$ of $18.5 \times 10^{-6} \mathrm{~kg} / \mathrm{ms}$.

"Glyecrol compositions given in parentheses as percent glyeerin (by mass) in water. 
Copyright (C)1997, American Institute of Aeronautics and Astronautics, Inc.

Table 2. Summary of properties of drops formed from the bag

\begin{tabular}{|c|c|c|c|c|c|c|c|c|c|}
\hline Liquid" & $\begin{array}{c}d_{n} \\
(\mu \mathrm{m})\end{array}$ & $\begin{array}{l}t / t^{*} \\
(-)\end{array}$ & $\begin{array}{c}\mathrm{h} \\
(\mu \mathrm{m})\end{array}$ & $\begin{array}{l}d_{\text {thb }} \\
(\mu \mathrm{m})\end{array}$ & $\begin{array}{l}\operatorname{SMD}_{\text {nd }} \\
(\mu \mathrm{m})\end{array}$ & $\begin{array}{l}\mathrm{h} / \mathrm{d}_{\mathrm{o}} \\
(\%)\end{array}$ & $\begin{array}{c}\mathrm{d}_{\mathrm{hdd}} / \mathrm{h} \\
(-)\end{array}$ & $\begin{array}{l}\mathrm{d}_{\mathrm{tdd}} / \mathrm{d}_{\mathrm{n}} \\
(\%)\end{array}$ & $\begin{array}{c}S M D_{\mathrm{ho}} / \mathrm{d}_{\mathrm{n}} \\
(\%)\end{array}$ \\
\hline Water & 620 & $3-4$ & 16.4 & 21.9 & 23.8 & 2.65 & 1.33 & 3.5 & 3.8 \\
\hline Glycerol (21\%) & 650 & $3-4$ & - & 22.1 & 23.9 & - & - & 3.8 & 3.5 \\
\hline Glycerol (42\%) & 650 & 3 & 23.2 & 25.8 & 28.9 & 3.57 & 1.12 & 4.0 & 4.5 \\
\hline Glyccrol (42\%) & 650 & $3-4$ & - & 29.6 & 33.2 & - & - & 4.6 & 5.1 \\
\hline Glyccrol $(63 \%)$ & 850 & $3-4$ & 35.4 & 42.1 & 48.8 & 4.16 & 1.17 & 4.9 & 5.2 \\
\hline
\end{tabular}

Results based on the propertics of the bag and the properties of drops formed by breakup of the bag during the bag breakup period for the test conditions summarized in Table 1.

"Glycerol compositions given in parentheses as percent glyccrin (by mass) in water.

\section{WATER, $W e=20, O h=0.0044$}

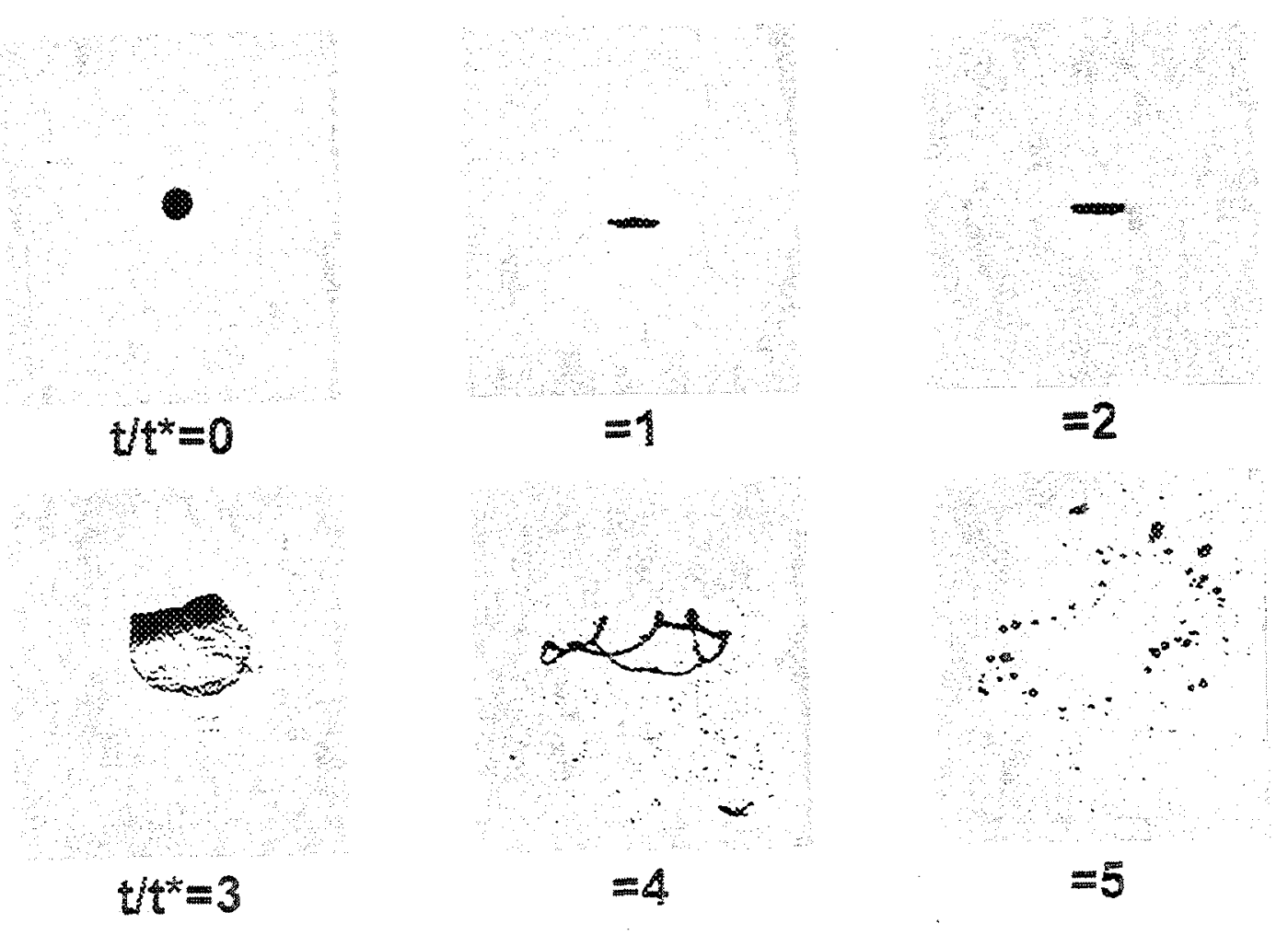

Fig. 1 Pulsed shadowgraph photo-graphs at various times during the bag breakup process: $\mathrm{We}=20$ and $\mathrm{Oh}$ $=0.0044$. 


\section{GLYCEROL (75\%), We=20, Oh=0.135}

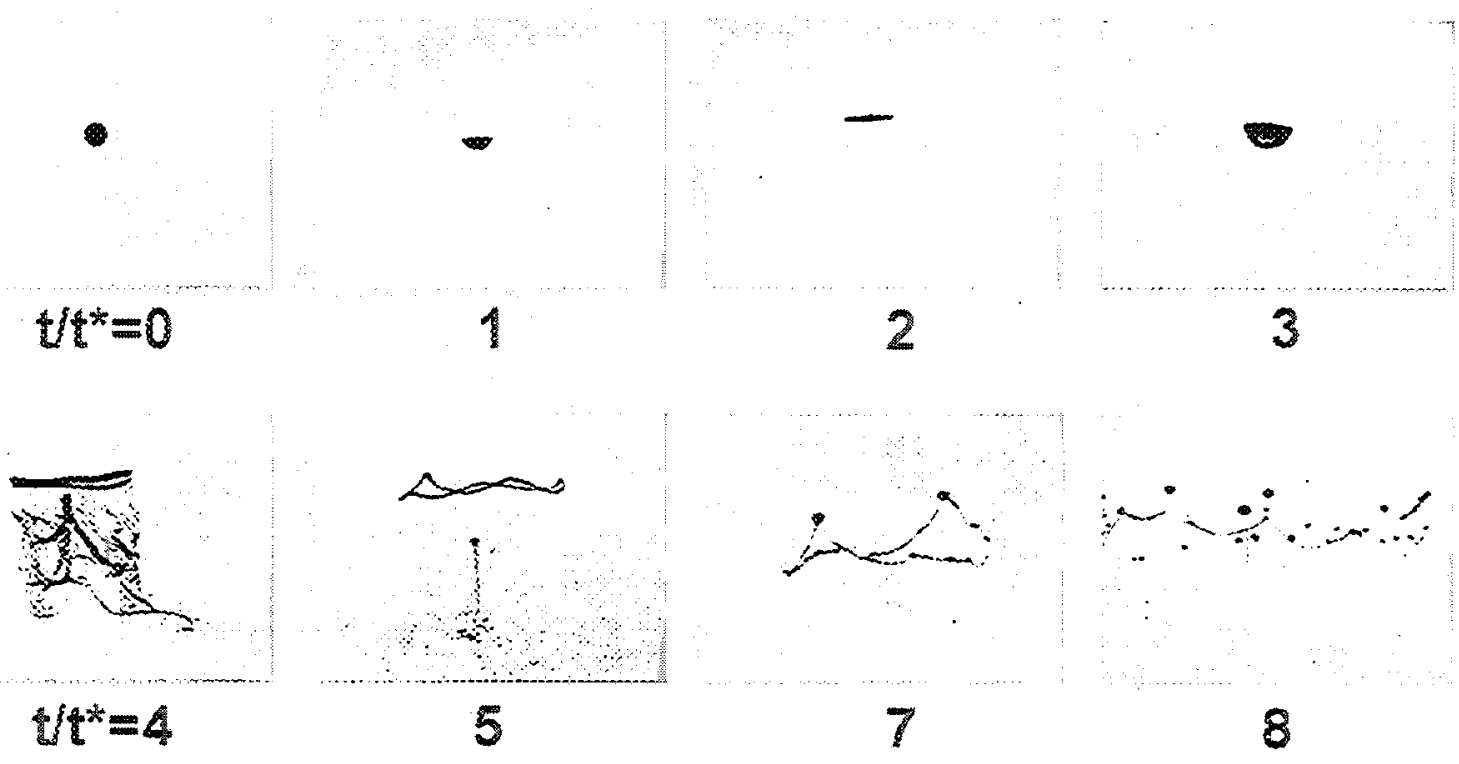

Fig. 2 Pulsed shadowgraph photo-graphs at various times during the bag breakup process: $\mathrm{We}=20$ and $\mathrm{Oh}$ $=0.135$.

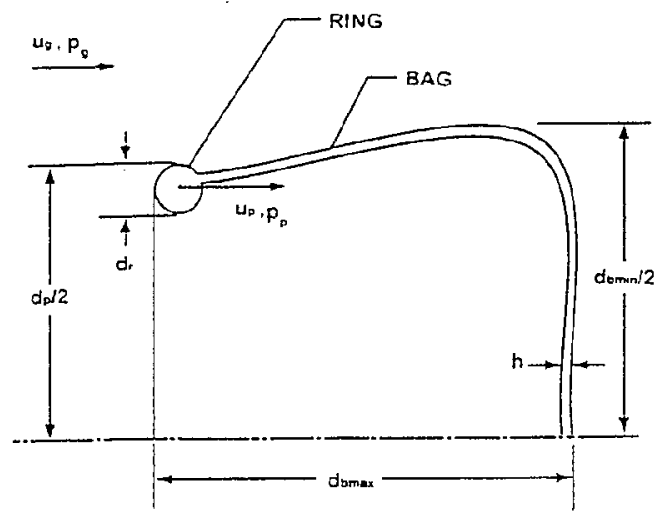

Fig. 3 Definition of bag breakup parameters. 


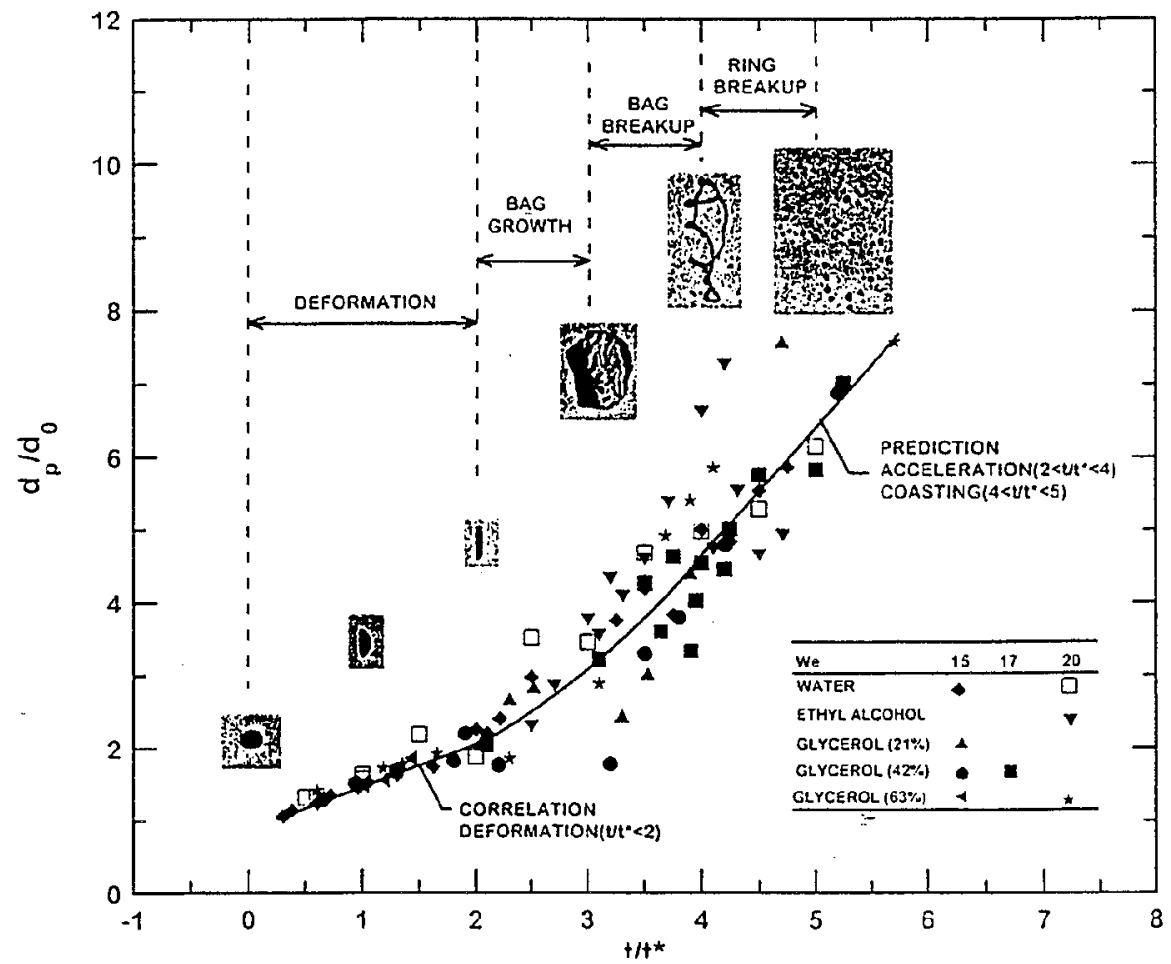

Fig. 4 Parent drop characteristic diameter, $d_{p}$, as a function of time during bag breakup.

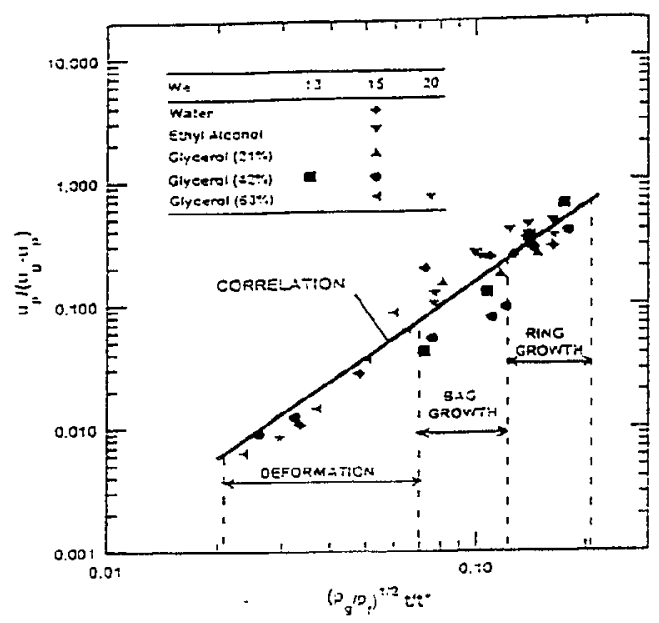

Fig. 5 Parent drop characteristic velocity, $u_{p}$, as a function of time during bag breakup.

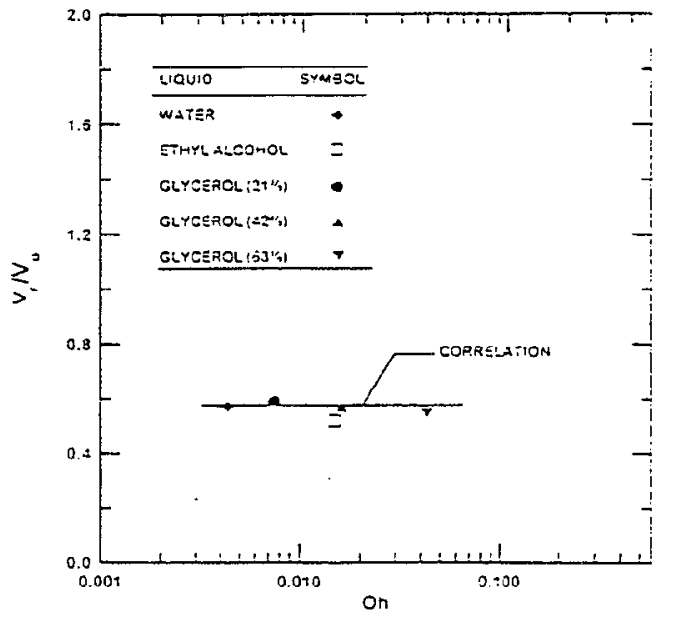

Fig. 6 Ratio of ring to original drop volume at the end of bag breakup. 


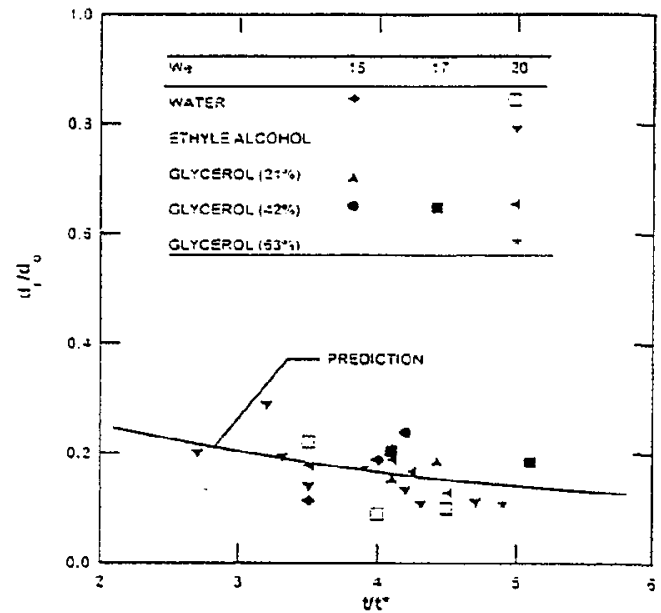

Fig. 7 Ratio of diameter of ring tube to original drop diameter as a function of time for bag breakup.

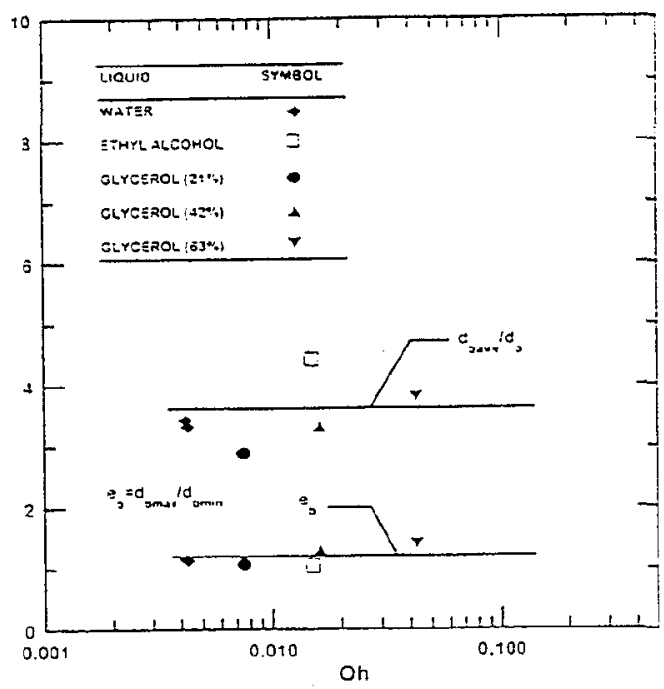

Fig. 9 Characteristic bag parameters, $d_{b a v e} / d_{o}$ and $e_{b}$, as a function of original drop Ohnesorge number for bag breakup.

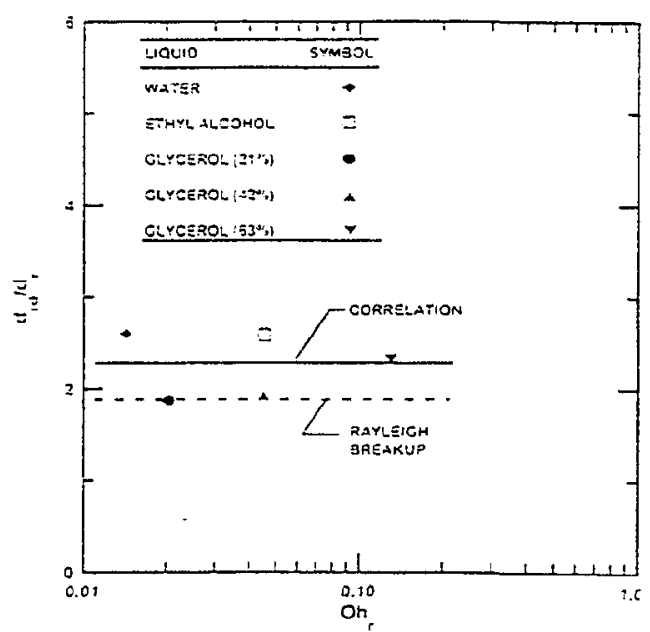

Fig. 8 Ratio of mean diameter of drops formed from ring and diameter of ring tube as a function of ring Ohnesorge number.

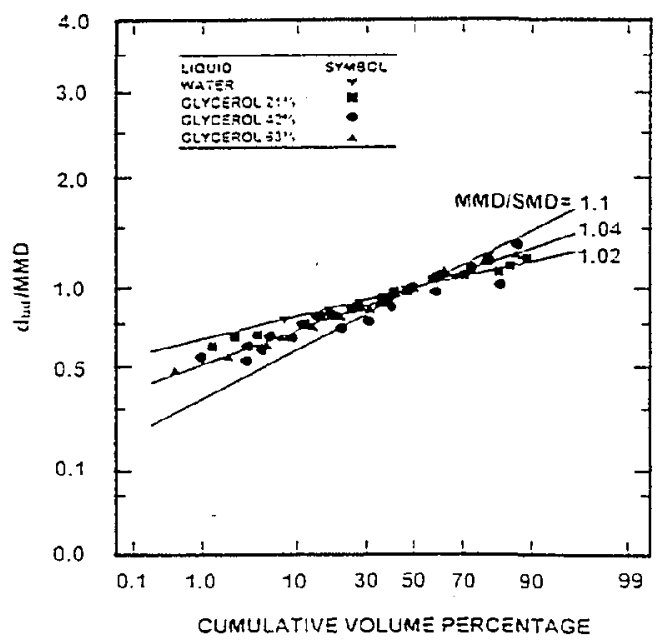

Fig. 10 Drop size distribution functions of drops formed from the bag after bag breakup. 


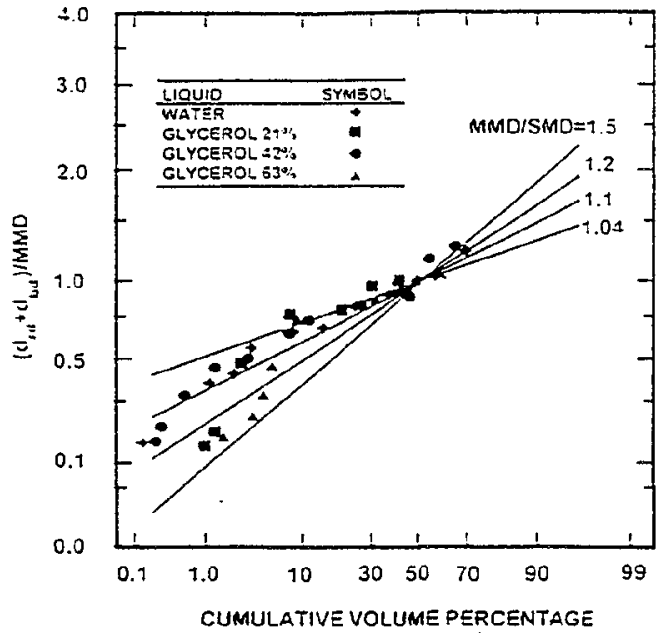

Fig. 11 Drop size distribution functions of drops formed from both the ring and the bag after bag breakup.

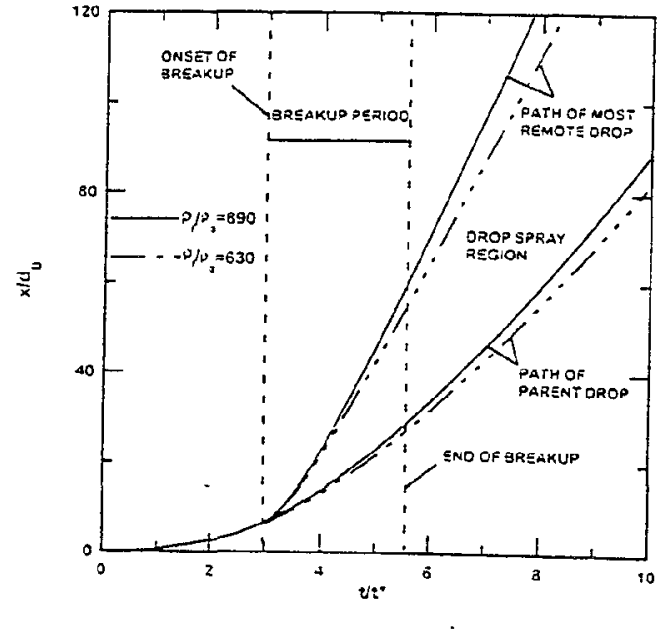

Fig. 12 Streamwise positions of the parent and the most remote drops as a function of time during bag breäkup. 